\title{
COMPREHENSIVE STUDY OF SILVER NANOPARTICLES OBTAINED FROM PINUS ROXBURGHII OBTAINED BY GREEN SYNTHESIS CHARACTERIZATION AND ANTIMICROBIAL APPLICATIONS.
}

\author{
Minakshi Gajanan Pawar \\ School of Life Sciences \\ SRTMU Nanded, Maharashtra, India
}

\author{
Ramjan M. Mulani \\ Director, School of Life Sciences \\ SRTMU Nanded, Maharashtra, India
}

\begin{abstract}
The increasing resistance of microorganisms against antibiotics threatens the microbiologist. Quantum dots are found to be an excellent antibacterial agent against $P$. aeruginosa. In the present study pinus roxburghii collected from the Mahabaleshwar region of Maharashtra, India.

The plants have been washed and dried in the shade for 10- 15 days. The $5 \mathrm{gm}$ dried plant powder was boiled in $100 \mathrm{ml}$ of double distilled water, whose extract is used for, The titration of $1 \mathbf{m m o l}$ silver nitrate solution. The change in color from pale yellow to red wine and, optical absorption peak in UV studies at $420 \mathrm{~nm}$ confirms existence of quantum dots. The morphology of the nanoparticles studied by FE-SEM analysis reveals, the particle size of $100 \pm 40 \mathrm{~nm}$. A good antimicrobial activity of obtained quantum dots, againstp. aeruginosa is evidenced with $17 \mathbf{~ m m}$ in diameter a zone of inhibition. As $p$ aeruginosa causes infection in wounds burns this study has recommended the use of quantum dots in ointments, lotions, and skin creams for early and safe recovery.
\end{abstract}

Key-words: Antibacterial activity, Escherichia coli. Quantum dots Pinusroxburghii Pseudomonas aeruginosa, Escherichia coli. Zone of inhibition

\section{INTRODUCTION}

Nanotechnology is the new technology which has entered in every sphere of life right from food to cloth. There are various nanoparticles like silver, gold, copper, titanium oxide, metal oxides, carbon nanotubes. The nanoparticles from transition metals are referred as a quantum dots. Synthesis of the quantum dots is gaining importance in many fields like biological sensor [1], antimicrobial applications [2],DNA sequencing, climate change, and contaminant control.[3], Clean water technology [5],information storage [6], and biomedical applications [7],clinical clothing biological sciences, pharmaceuticals, and medicine. There are various methods for the synthesis of nanomaterials via chemical method, physical method and biological methods. The biological method the biological method Includes use of biological entities like microorganism, [11], like bacteria [12], fungi. [13], the plants, [15] and the bimolecular like honey polysaccharides like glucose etc. the plant has gained importance due to fact that they are available numerous easily available and non toxic do not produce any gases heat and radiations or a noise.. First of all in 2003 the green synthesis was demonstrated by the toad.

As the plant extract is rich source of different types of reducing and capping agent. So this is the only method which can produced nanoparticles having various morphology. Until now the large number of plants is used in green synthesis. Pelargonium [15] Nillumbik nucifera [16]. The herb chirr pine is belongs to the Piniaceae family whose botanical name is pinusroxburghii. The plant is an evergreen Tree the high about 131 feet near about 40 meter. The tan. It is a green dye obtained from the needle of the plants. The Seeds are eaten as-raw or cooked is eaten as emergency food. A reasonable size the seed is up to $11 \mathrm{~mm}$ long. A sweet edible manna exudes from the bark and twigs. It is actually a gum. A vanillin flavoring is obtained as a byproduct of other resins that are released from the pulpwood.

The seeds of the chirr plants are also useful they are eaten as a raw or cocked and used as a food. In the present study we have synthesizes quantum dots.

\section{MATERIAL AND METHODS}

Preparation of leaf extract:

Plants leaves were collected from Mahabaleshwer region in the month of February. The plant was authenticated by Prof.R.M.Mulani. Director of school of life sciences. Swami Marathwada University, Nanded. The plants were washed with $70(\mathrm{v} / \mathrm{v})$ ethanol (1 minute). Later these were washed three times with double distilled water. Plants were air -dried for 15 days in Laboratory the needle were ground to a fine powder. 25 gram of needle powder was boiled in $100 \mathrm{ml}$ of double distilled water in a $250 \mathrm{ml}$ of Erlenmeyer flask. The mixture were filtered through Whitman's filter paper no .4. The filtrates were stored in refrigerator for further use at $4^{\circ} \mathrm{C}$. 


\section{Biosynthesis of quantum dots.}

Silver nitrate was purchased from Sigma Aldrich, USA. One mill molar silver nitrate solution were prepared $.100 \mathrm{ml}$ silver nitrate solution was triturated against needle extract. The color changes from the pale yellow to dark red wine. The formation of quantum dots was confirmed from the ultra violet color spectroscopy.

\section{The Characterization of synthesized quantum dots Ultraviolet -visible (U V-VIS) Spectroscopy}

Silver is a noble silver metal which that posses strong absorption in the Vies region with the maximum in the range $400-450 \mathrm{~nm}$. The quantum dots shows the surface plasma resonance phenomenon. This phenomenon is attributed to collective oscillation of free conduction electromagnetic field in silver metallic nanoparticles. There is change in pale yellow to red wine. The observation of an absorption band in 410-450nm.Theirfor U. V.-vies spectroscopy is, usually is the first confirmatory test to check the formation of quantum dots.,
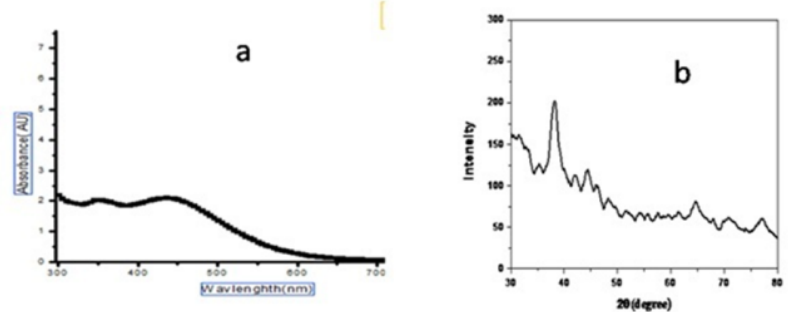

\section{Figure 1a) UV Analysis quantum dots of Pinus roxburghii b) XRD of quantum dots of Pinus roxburghii}

the SPR band can give useful information about the size, and shape of the synthesized nanoparticles the increase in size causes the increase in maximum wavelength (red shift), and the reduction in particle size leads to decrease in maximum wavelength (blue shift). In anisotropic nanoparticles the asymmetrical and broad SPR is observed the fig1a) shows the $u v$ analysis of the

Quantum dots the peak was observed at $420 \mathrm{~nm}$. The quantum dots were stable for about six months. XRDthis is the technique is used to obtain the structural information about crystalline quantum dots and confirms the formation of zero Valeant nanoparticles. Since every crystalline materials by comparing the obtained pattern with the reference library if the nanoparticles are produced in an amorphous structure, no diffraction peak is observed the broadening of the peak XRD confirms the formation of particles in anodizing [10]. fig1 b) shows the XRD analysis of the quantum dots...

The XRD pattern of synthesized nanoparticles was shown in fig $1 \mathrm{~b}$ ), the spectra data reveals the occurrence of four significant peaks observed at 38, 44, 64and 77. These peaks of various intensities are seen in the fig-1b) these peaks are related to the Braggs reflections of (111), (200), (222), (311) and (222) plans of face -centered cubic phase which are closely related to standard of joint committee on powder diffraction standard JCPDS. The sharp peaks confirm the synthesizes quantum dots are crystalline in nature. Nanoparticles were centrifuged at $18000 \mathrm{rpm}$ for 20 minutes. The pellets are collected washed with double distilled water three times transferred to Petri-plats dried in a hot air oven. It is father used for characterization

\section{ANTIBACTERIAL ASSAY -}

Antibacterial activity of synthesized quantum dots was determined using well diffusion method. The overnight incubated bacterial cultures were harvested. The nutrient agar plates were made by pouring the $20 \mathrm{ml}$ sterilized nutrient medium on sterilized petri plate in aseptic condition. The plate is allowed to solidify in the $u v$ chamber of laminar air flow. A loopful of active bacterial culture is spread on the nutrient agar plate. The nutrient agar plate is divided into three parts, by glass marker. With the help of cork borer. In one well one millimolar silver nitrate solution is dropped with the help of micropipette. In the next well nanoparticle solution were dropped. The standard antibiotic disc (Hymenia) is placed as the reference.

\section{Scanning electron microscope analysis FESEM-}

For the FESEM analysis the sample should be in powder form the colloidal solution of silver nanoparticles is centrifuged at $15000 \mathrm{rpm}$ for 20 minutes. The pellet were collected and washed with Doubled distilled water three times the pellet were collected in large glass dish it is kept in hot air oven for 24 hours at $100^{\circ}$ centigrade. The thin layer of quantum dots is observed on the glass of a sheet. It is scrubbed with the help of the blade. Now the powder is collected and stored in screw cap tube collecting Tubes at the time of FESEM analysis the powder is mounted on a sample holder followed by coating with conducting metal, such as gold, and fixed by using a tape.
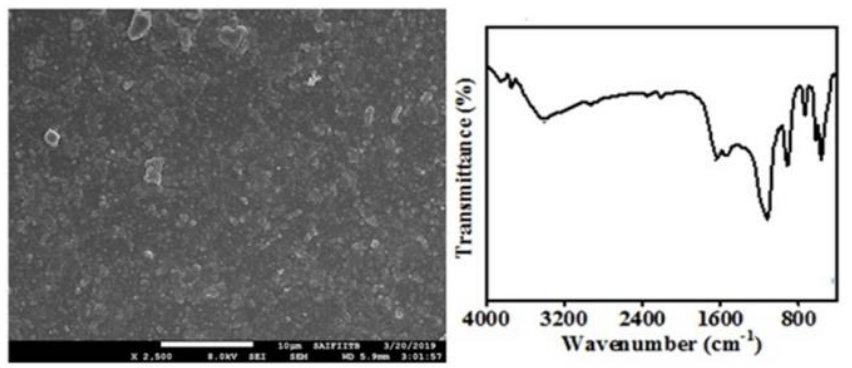

Figure 2a) FE-SEM image of quantum dots of needle of Pinus roxburghii b) FTIR of quantum dots of Pinus roxburghii

This is the technique to obtain an image of nanomaterials in SEM, Instrument a fine electron probe, formed by a small electron source, and is scanned over a specimen in a two dimensional raster. Signals generated from the specimen are detected, amplified and, used to modulate the brightness of a second electron beam that is scanned synchronously with the SEM Electron probe across a cathode ray tube (CRT) display. Therefore a specimen image is mapped onto the CRT display for observation. Since SEM is serial recording instead of parallel recording system, the while process of generating a SEM Image could be slower than that in a TEM. The surface morphology of quantum dots was confirmed from images image no 2a) As depicted in SEM structure revels a random distribution of 
quantum dots in an aggregated form, making it complicated to find the precise size and shape of individual quantum dots.

\section{High Resolution transmission Electron Microscope-}

HRTEM-it is one of the most reliable techniques to study the structure of nano-materials. HRTEM refers to imaging in which lattice fringes (i.e. Crystallographic planes) are observed or atomic resolution is achieved. HRTEM image are formed from a number of diffracted beams. This multi- beam approach is known as phasecontrast imaging, and is necessary to construct an image of the crystal lattice. HRTEM image Provides access to much information about the sample, such as analyzing crystalline defects and interfaces at the atomic scale, and observing and verifying devices, multilayer nanocrystals and nanostructures. Crystallite size and morphology of synthesis quantum dots form confirmed the TEM in the figure. 3a) and $3 b$.
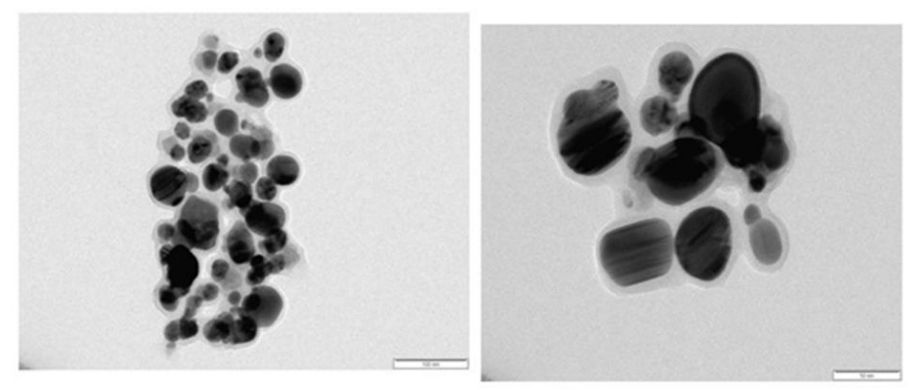

\section{Figure 3a) HR-TEM Image of quantum dots of Pinus roxburghii b) HR-TEM of quantum dots of Pinus roxburghii.}

These images demonstrated that that self gown nanoparticle structures. Average diameter of these quantum dots was calculated from selecting the particle in random fields of view the average particle size40-100nm.Crystallite size and, morphology of synthesis quantum dots were conformed from the high resolution transmission electron microscope (TEM ) in the figure. 3 a) and $3 b$ )These images demonstrated that as a self gown nanoparticle structure. Average diameter of these quantum dots was calculated from selecting the particle in random fields of view the average particle size.40-100nm.Image also confirm the irregular surfaces both images corroborated the irregular surfaces from 2-d lattices in the distance, which are good argument with interlayer reflection of () in the JCPDS index card of $40-100 \mathrm{~nm}$ the average particle size of quantum dots obtained from HR-TEM

Provides access to much information about the sample, such as analyzing crystalline defects and interfaces at the atomic scale, and observing and verifying devices, multilayer nano crystals and nanostructures. Transmission electron e microscope analysis

\section{RESULT AND DISCUSSION}

The result of UV spectroscopy, When red wine colored quantum dots were analyses in UV. Spectroscopy. The peak was observed at $420 \mathrm{~nm}$. Which confirmed the formation of quantum dots? Chemically it is bio-reduction of silver nitrate solution. The reduction takes place due to the functional groups present in the plant extract. In plants as, the secondary metabolites are present in plants such as glycosides alkaloids, saponins, flavonoids, tannins and amino acids These functional groups may act as a reducing agents and the quantum dots are formed. The formed nanoparticles are stable for about six months.

Antibacterial activity of quantum dots of $P$. roxburghii against $E$. coli.

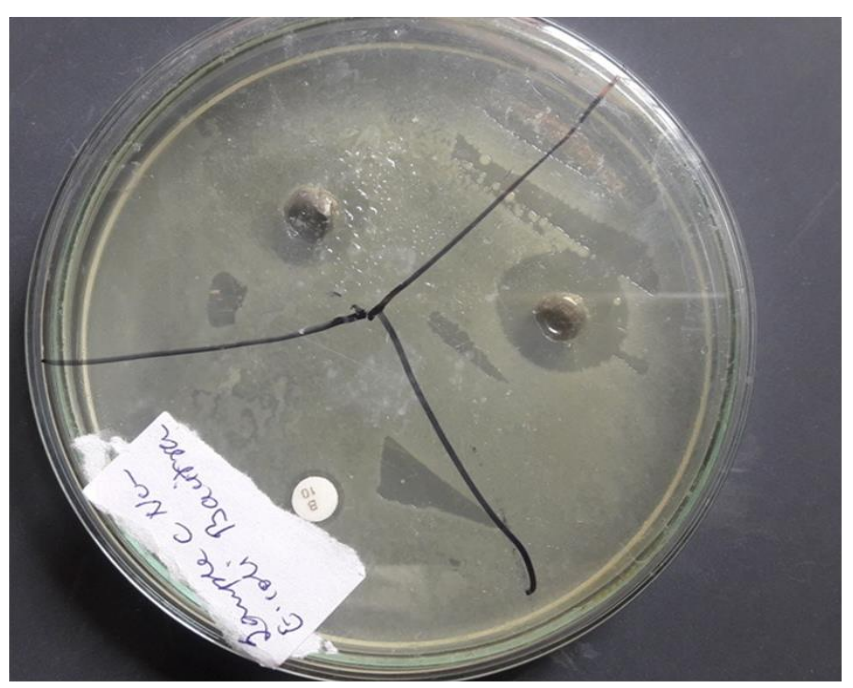

Figure 5) antibacterial activity of Escherichia coli against

Quantum dots obtained by needle of Pinus Roxburgï

Mechanism of antibacterial activity. The antibacterial study shoes that $e$ coli is a gram negative bacteria showing good antibacterial activity the zone of inhibition is around $23 \mathrm{~mm}$ in diameter. The chemical composition of cell wall of gram negative $E$ coli is responsible for the antibacterial activity of gram- negative bacteria. As E coli is one of the microorganisms responsible for water contamination and causes water burn diseases like amoebiasis in rainy season. As newly synthesized quantum dots are showing excellent antibacterial activity against E.coli. This study recommends the use of quantum dots to se in water purification process. 


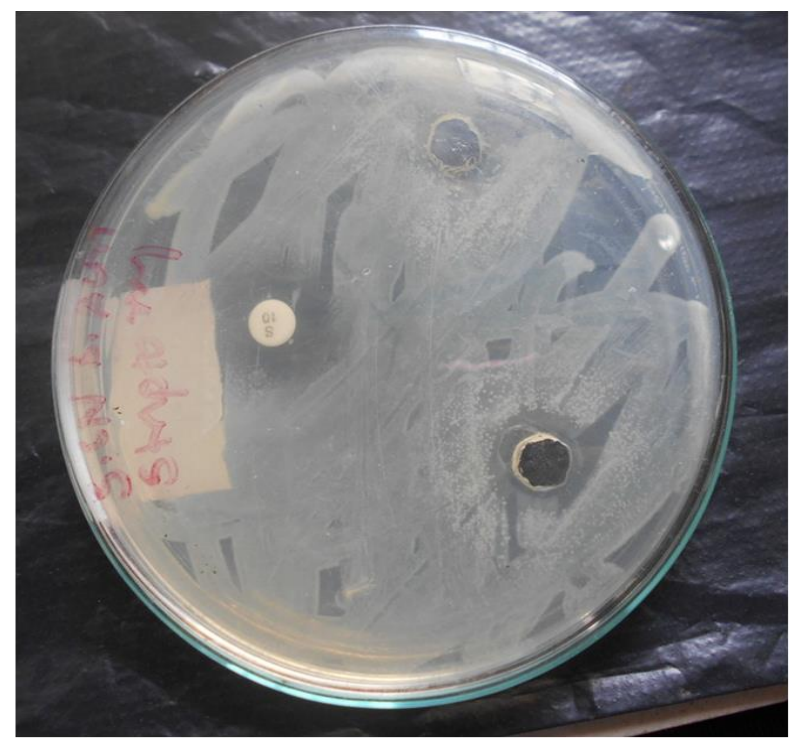

Figure 5 Antimicrobial activities of $P$. aeruginosa against quantum dots obtained from needle of Pinus roxburgi

An excellent antibacterial activity was shown by pseudomonas aeruginosa. It is a gram negative bacteria which causes urinary tract infection, pulmonary infection soft tissue infections epsis, bone and joint infection endocarditis. This study may suggest that quantum dots are good antibacterial agent which can be used in ointments, lotions, and skin creams it can be used in cementing material of joints, teeth wound and burn healing lotions.

\section{CONCLUSION}

Green synthesis of quantum dots was one of the important methods of synthesis of nanoparticles it is ecofriendly, easy to scale up. One of the plus points of this method is that it does not give poisonous chemicals, product, does not emit light, heat, or gases. In this process there is no any capping or stabilisizing agent plant extract itself act as capping and stabilizing agent the major drawback of this process is that one cannot obtained the particles of uniform size. This is due to the fact that Pinusroxburghii extract contains bimolecular such as turpentineresins alkaloids etc. Among these bimolecular which is acting as a reducing agent and which is acting as stabilizing agent is not clear. the difference in nanoparticle may be found because geographical area, environmental conditions, and seasons are responsible for the different photochemical composition in plants so that from same plants of different area nanoparticle are different `sizes.

As we have synthesizes quantum dots which is shown The UV. peak at $420 \mathrm{~nm}$ the surface morphology is shown by FESEM analysis shows non uniform sizes of quantum dots.

The TEM image shoes the variety of nanoparticles having sizes $40-100 \mathrm{~nm}$ in size. The antibacterial activities were studied by gram negative bacteria. it shows good antibacterial act by pseudomonas aeruginosa as e well as E. Coli. This study may suggest that quantum dots are good antibacterial agent which can be used in ointments, lotions, and, skin. It can be used in cementing material of joints, teeth, wound and burn healing lotions. These quantum dots can be used in water purifier.

\section{REFERANCES}

1. Chad A. Marking, Robert L. Let singer, Robert C. Music \& James J. Storhoff, "DNA-based method for rationally assembling nanoparticles into macroscopic materials," nature, 386-607.1996.

2. Savithramma N, Rao ML, Rukmini K, Devi PS., " Antimicrobial activity of quantum dots synthesized by using medicinal plants, " Int J ChemTech Res.,3:13941402. 2011.

3. Cao Y, Jin R, Mirkin CA. , " DNA-modified core-shell $\mathrm{Ag} / \mathrm{Au}$ nanoparticles," J Am Chem Soc. 123:79617962.2001.

4. Shan G, Surampalli RY, Tyagi RD, Zhang TC. 2009., " Nanomaterials for environmental burden reduction, waste treatment, and nonpoint source pollution control: a review.", Front Environ Sci Eng China,. 3:249-264. 2009.

5. Z€ach M, H€agglund C, Chakarov D, Kasemo B ,". Nanoscience and nanotechnology for advanced energy systems., " Curr Opin Solid State Mater Sci.,10:132143. 2006

6. Caruthers SD, Wickline SA, Lanza GM.,"Nano technological applications in medicine", . CurrOpinBiotechnol. 18:26-30. 2007.

7. Hullmann A.2007." Measuring and assessing the development of nanotechnology," ,Scientometric,. 70:739-758. 2007

8. Noruzi M, Zare D, Davoodi D 2012, " A rapid biosynthesis route for the preparation of gold nanoparticles by aqueous extract of cypress leaves at room temperature, ". SpectrochimActa Part A Mol Biomol Spectrosc 94:84-88.2012.

9. Vilchis-Nestor AR, Sánchez-Mendieta V, CamachoLópez MA, Gómez-Espinosa RM, Camacho-Lopez MA, Arenas-Alatorre JA (2008) , "Solvent less synthesis and optical properties of $\mathrm{Au}$ and $\mathrm{Ag}$ nanoparticles using Camellia sinensis extract," Mater Lett, 62:3103-3105 .2008

10. Das RK, Borthakur BB, Bora U, " Green synthesis of gold nanoparticles using ethanolic leaf extract of Centellaasiatica, "Mater Lett 64:1445-1447 .2010.

11. Hebbalalu D, Lalley J, Nadagouda MN, Varma RS, " Greener techniques for the synthesis of quantum dots using plant extracts, enzymes, bacteria, biodegradable polymers, and microwaves, ". ACS Sustainable Chem. Eng .2013,

12. Vijayaraghavan K, Nalini SP, Prakash NU, Madhankumar D:,"One step green synthesis of silver nano/micro particles using extract of Trachyspermumammi and Papaversomniferum.",Colloids Surf Biointerfaces 2012,94:14-17. 10.1016/j.colsurfb.2012.01.0262012 
13. Srivastava P, Bragança J, Ramanan SR, Kowshik M:," Synthesis of quantum dotss using haloarchaeal isolate Halococcussalifodinae BK3.,"Extremophiles , 17: 821831.

14. 201310.1007/s00792-013-0563-3

15. Huang J, Li Q, Sun D, Lu Y, Su Y, Yang X, Wang H, Wang Y, Shao W, He N, Hong J, Chen C: "Biosynthesis of silver and gold Nanoparticles by novel sundried Cinnamomumcamphor leaf,". Nanotechnology
2007, 18: 105104-105114. 10.1088/09574484/18/10/105104

16. Shankar SS, Ahmad A, and Satyr M, "Geranium leaf assisted biosynthesis of quantum dots, ". BiotechnolPrig 2003, 19: 1627-1631. 10.1021/bp034070wn

17. Metal AK, Christi Y, Bannered UC:,"Synthesis of metallic nanoparticles using plant extracts, Biotechnol Adv 2013, 31: 346-356. 